\title{
Artrodese lombar intersomática anterior multinível combinada com estabilização posterior em discopatia-Análise clínico-funcional prospectiva*
}

\section{Multilevel Anterior Lumbar Interbody Fusion Combined with Posterior Stabilization in Lumbar Disc Disease-Prospective Analysis of Clinical and Functional Outcomes}

\author{
Diogo Lino Moura ${ }^{1,2}$ David Lawrence ${ }^{2}$ Josué Pereira Gabriel ${ }^{2}$

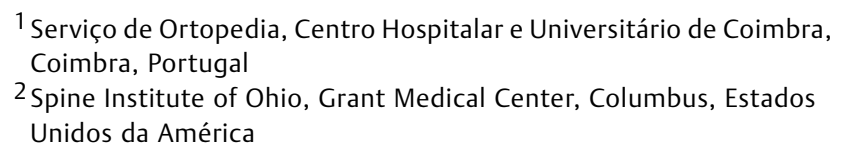

Address for correspondence Diogo Lino Moura, Serviço de Ortopedia, Centro Hospitalar e Universitário de Coimbra, Coimbra, Portugal (e-mail: dflmoura@gmail.com).

Rev Bras Ortop 2019;54:140-148.

\begin{abstract}
Resumo
Palavras-chave

- vértebras lombares/ cirurgia

- fusão vertebral

- estudos prospectivos

- escoliose/cirurgia

- fatores de risco

Objetivo Estudo prospectivo controlado em pacientes com discopatia degenerativa submetidos a artrodese intersomática lombar anterior instrumentada combinada com estabilização posterior.

Métodos Amostra com 64 pacientes consecutivos operados pelos mesmos cirurgiões ao longo de quatro anos. Metade das artrodeses intersomática lombar anterior foi efetuada em dois níveis, 43,8\% em três níveis e 6,25\% em um nível. Foram usadas caixas intersomáticas com parafusos integrados preenchidas com matriz óssea e proteína morfogenética óssea 2.

Resultados Metade da amostra apresentava cirurgias prévias à coluna lombar, 75\% listeses degenerativas associadas e $62,5 \%$ patologia compressiva posterior da coluna lombar. Aproximadamente $56 \%$ da amostra apresentavam pelo menos um fator de risco de não união da artrodese. O índice Oswestry passou de 71,81 \pm 7,22 no pré-operatório para 24,75 \pm 7,82 na avaliação no fim do tempo de seguimento, enquanto a escala visual analógica da dor passou de 7,88 $\pm 0,70$ para $2,44 \pm 0,87$ ( $p<0,001$ ). A melhoria clínicofuncional foi crescente de acordo com a intervenção num número superior de níveis, o que comprova a eficácia da artrodese intersomática lombar anterior multinível, aplicada em $93,75 \%$ da amostra. A taxa global de complicações foi de 7,82\% e de complicações major de $0 \%$. Não se identificou qualquer caso de não união.

Conclusão A artrodese intersomática lombar anterior instrumentada combinada com estabilização posterior é uma opção de sucesso na discopatia degenerativa uni ou multinível dos segmentos de L3 a S1, mesmo em presença significativa de fatores de
\end{abstract}

\footnotetext{
* Trabalho desenvolvido no Serviço de Ortopedia, Centro Hospitalar e Universitário de Coimbra, Coimbra, Portugal. Publicado originalmente por Elsevier Ltda.
}

received

October 21, 2017

accepted

November 28, 2017

published online

April 15, 2019
DOI https://doi.org/

10.1016/j.rbo.2017.11.006. ISSN $0102-3616$.
Copyright $(2019$ by Sociedade Brasileira License terms de Ortopedia e Traumatologia. Published by Thieme Revnter Publicações Ltda, Rio de Janeiro, Brazil 


\section{Abstract}

\section{Keywords}

- lumbar vertebrae/ surgery

- spinal fusion

- prospective studies

- scoliosis/surgery

- risk factors risco de não união e cirurgias prévias da coluna lombar, garante resultados clínicofuncionais e radiográficos muito satisfatórios e reduzida taxa de complicações em médio prazo.

Objective This was a prospective controlled study with lumbar degenerative disc disease patients submitted to instrumented anterior lumbar interbody fusion (ALIF) combined with posterior stabilization.

Methods A sample with 64 consecutive patients was operated by the same surgeons over 4 years. Half of the ALIFs occurred at 2 levels, $43.8 \%$ at 3 levels, and $6.25 \%$ at 1 level. Interbody cages with integrated screws, filled with bone matrix and bone morphogenetic protein 2, were used.

Results Half of the patients had undergone previous lumbar spine surgeries, 75\% presented with associated degenerative listhesis, and $62.5 \%$ had posterior lumbar compression disease. Approximately $56 \%$ of the sample had at least 1 risk factor for nonunion. The Oswestry index changed from $71.81 \pm 7.22$ at the preoperative assessment to $24.75 \pm 7.82$ at the final follow-up evaluation, while the visual analogue pain scale changed from $7.88 \pm 0.70$ to $2.44 \pm 0.87(p<0.001)$. Clinical and functional improvements increased with the number of operated levels, proving the efficacy of multilevel ALIF, performed in $93.75 \%$ of the sample. The global complication rate was of $7.82 \%$, with no major complications. No cases of nonunion were observed. Conclusion Instrumented ALIF combined with posterior stabilization is a successful option for uni- and multilevel degenerative disc disease of the L3 to S1 segments, even in the significant presence of risk factors for nonunion and of previous lumbar surgeries, assuring very satisfactory clinical-functional and radiographic outcomes with a low medium-term complication rate.

\section{Introdução}

A artrodese intersomática lombar anterior (ALIF) é uma das opções para o tratamento da discopatia e espondilolistese degenerativas da coluna lombar nos níveis L3-L4, L4-L5 e L5-S1, patologias em crescendo na sociedade atual. ${ }^{1} \mathrm{Em}$ teoria, a artrodese intersomática efetuada por via anterior tem vantagens biomecânicas e em termos de morbilidade face às vias posteriores, oblíquas ou laterais. ${ }^{2,3} \mathrm{~A}$ via anterior da coluna lombar permite uma melhor exposição do espaço discal e aplicação duma caixa intersomática de maior dimensão, restaura assim de maneira mais eficaz a altura do espaço intervertebral, a lordose lombar, o balanço sagital e a distribuição fisiológica de forças sobre as colunas anterior e média de Dennis (80\% das forças compressivas axiais ocorrem nas colunas anterior e média), permite assim em teoria diminuir o risco de doença do disco adjacente e a necessidade de futuras intervenções cirúrgicas. Esses fatores permitem também em teoria aumentar o potencial de fusão intersomática, na medida em que a caixa fica mais sujeita a forças de compressão na coluna anterior de Dennis, existe um estímulo mais eficaz à fusão óssea. ${ }^{1-6}$ Além disso, a posição anterior da caixa corresponde à região mais vascularizada do corpo vertebral, o que estimula a artrodese. ${ }^{4,5} \mathrm{~A}$ discectomia mais eficaz sob visualização direta através dum espaço mais amplo permite que existam menos resíduos discais que se possam interpor e desfavorecer a fusão intersomática em comparação com outras vias, garante uma maior área para artrodese. O maior espaçamento intersomático proporcionado pela caixa de maior dimensão permite também aumentar significativamente a altura dos orifícios intervertebrais, diminui assim de forma eficaz o conito com as raízes raquidianas e a sintomatologia. ${ }^{3,7,8}$ Em termos de morbilidade, ao contrário das vias posteriores, que implicam disseção extensa dos músculos paravertebrais, e da via lateral, que implica atravessar o psoas, a via anterior da coluna lombar não interfere com qualquer músculo da coluna vertebral e não inclui desinserções musculares. Assim, é uma via em teoria menos sangrativa, o que pode permitir uma recuperação mais rápida em termos álgicos (com menor necessidade de analgésicos) e funcionais no pós-operatório (com menor tempo de internamento) e uma coluna vertebral precocemente mais estável por não interferir com a musculatura estabilizadora. ${ }^{3,5,6,8-10}$ Além disso, a via anterior também não implica remoção de elementos posteriores da coluna vertebral, nem entrada no canal medular ou manipulação de raízes raquidianas para acesso ao espaço discal, diminui assim o risco de lesão iatrogênica e de complicações nessas importantes estruturas em comparação com as vias posteriores. ${ }^{3,6,8}$

Apesar dessas vantagens teóricas e de a ALIF estar descrita desde a década de 1930 para o tratamento de várias patologias 
da coluna lombar, as suas indicações exatas e as vantagens claras permanecem por comprovar. ${ }^{1,2,8,11}$

Frequentemente, o receio da lesão iatrogênica e potencialmente fatal dos grandes vasos, bem como a reduzida experiência da cirurgia ortopédica na via de abordagem anterior da coluna lombar, tem afastado muitos cirurgiões dessa via de fusão intersomática. Atualmente os estudos prospectivos de grande dimensão sobre ALIF continuam a ser limitados, em muitos centros a ALIF é preterida face a fusões intersomáticas por via posterior, oblíqua ou lateral direta.

\section{Material e métodos}

Estudo prospetivo controlado em 64 pacientes consecutivos ao longo de quatro anos com discopatia degenerativa lombar submetidos a ALIF instrumentada combinada com estabilização posterior, todos operados pelos mesmos cirurgiões e de acordo com o mesmo protocolo terapêutico.

O tempo médio de seguimento foi de 27,64 \pm 11 meses (tempo mínimo de 12 e intervalo 12-48). Todos os pacientes cumpriram um período de tratamento conservador, inclusive controle sintomático e fisioterapia, até se avançar com a intervenção cirúrgica. Os pacientes foram estudados quanto ao diagnóstico, à sintomatologia, os fatores de risco de não união (obesidade, tabagismo, diabetes mellitus, cirurgia multinível, ${ }^{2,12,13}$ características da intervenção cirúrgica, do internamento e complicações correspondentes à ALIF. Para análise clínico-funcional aplicaram-se o índice de incapacidade Oswestry ${ }^{14}$ e a escala visual analógica da dor, ${ }^{15}$ compararam-se valores do pré-operatório com os obtidos na avaliação final do tempo de seguimento de cada paciente. A análise radiográfica incluiu pesquisa de sinais de migração do implante, de falência da sua fixação e presença de osteólise peri-implante (sinais indiretos de não união). As variáveis foram tratadas estatisticamente com recurso ao programa SPSS Statistics for Windows, Version 23.0 (IBM Corp., Armonk, NY). O teste de normalidade Shapiro-Wilk identificou distribuições assimétricas das variáveis, pelo que foram aplicados testes estatísticos não paramétricos. Os valores de $p$ inferiores a 0,05 foram considerados estatisticamente significativos. 0 presente estudo foi aprovado pela instituição em causa.

\section{Protocolo terapêutico}

Os detalhes da cirurgia ALIF estão descritos na - Tabela 1. A via de abordagem anterior da coluna lombar é efetuada e encerrada por cirurgião vascular experiente nessa abordagem. A ALIF é complementada num segundo tempo operatório e num segundo internamento com fixação transpedicular posterior percutânea (artrodese de 270。) ou, se existir necessidade de lamino-foraminectomia descompressiva, com artrodese posterolateral (artrodese de 360。 ou circunferencial). Em todos os procedimentos cirúrgicos é efetuada neuromonitoração intraoperatória. Todos os pacientes são avaliados clinica e radiograficamente na primeira semana, às seis semanas, aos três meses, seis meses e um ano de pós-operatório, seguem-se consultas anuais (-Figs. 1 e 2). Não fazemos por sistema tomografia computadorizada para confirmar presença de união.

\section{Resultados}

A maioria da amostra $(75 \%, \mathrm{n}=48)$ é do sexo masculino, média de 53,63 \pm 9,47 anos (intervalo 29-69). Três quartos da amostra $(\mathrm{n}=48)$ apresentavam listeses degenerativas associadas à discopatia e $62,5 \%(n=40)$ apresentavam patologia compressiva posterior da coluna lombar concomitante. Vinte pacientes foram operados já com algum grau de déficit neurológico, que foi desde diminuição da força muscular a situações de pé pendente. Os sintomas predominantes foram radiculopatia lombossacra $(96,88 \%, \mathrm{n}=62)$ e dor lombar axial $(65,63 \%, \mathrm{n}=42)$. Metade dos pacientes operados apresentava intervenções prévias à coluna lombar, que se dividiam entre artrodeses intersomáticas posteriores (PLIF) $(\mathrm{n}=8)$ e transforaminais (TLIF) $(\mathrm{n}=4)$ de um nível, laminectomias $(n=12)$ e micro-discectomias $(n=8)$ (-Fig. 3 ). Todos os PLIFs operados eram situações de não união, enquanto nos pacientes com TLIFs eram casos de doença do disco adjacente. A fixação posterior foi mantida nos casos de PLIFs e TLIFs prévios. Mais de metade dos pacientes operados (56,25\%; $\mathrm{n}=36$ ) apresentava pelo menos um fator de risco de não união da artrodese, cuja distribuição está demonstrada na - Fig. 4. A distribuição dos segmentos operados está ilustrada na -Fig. 5, a ALIF foi multinível em $93,75 \%$ dos casos. As dimensões das caixas intersomáticas mais frequentemente usadas de acordo com o segmento

Tabela 1 Detalhes da cirurgia ALIF efetuada para todos os pacientes da amostra

\begin{tabular}{|l|l|}
\hline Protocolo terapêutico & Via anterior abdominal retroperitoneal da coluna lombar \\
\hline Via de abordagem & $\begin{array}{l}\text { De acordo com a presença de discopatia degenerativa, listese e necessidade de restauro da } \\
\text { lordose lombar }\end{array}$ \\
\hline Caixas intersomáticas & $\begin{array}{l}\text { Anatômicas, lordóticas, com superfície ranhurada e opc, ão de integrar parafusos através do } \\
\text { implante. O material constituint das caixas é o PEEK }\end{array}$ \\
\hline Preenchimento das caixas & $\begin{array}{l}\text { Matriz óssea desmineralizada e placa de colagênio absorvível com recombinante humano de } \\
\text { proteína morfogenética óssea 2 (BMP-2) (InFUSE } 囚)\end{array}$ \\
\hline Fixação das caixas & Fixas com 3 parafusos, dois no corpo vertebral inferior e um no superior. \\
\hline
\end{tabular}

Abreviações: ALIF, artrodese intersomática lombar anterior; PEEK, poliéter-éter-cetona. 

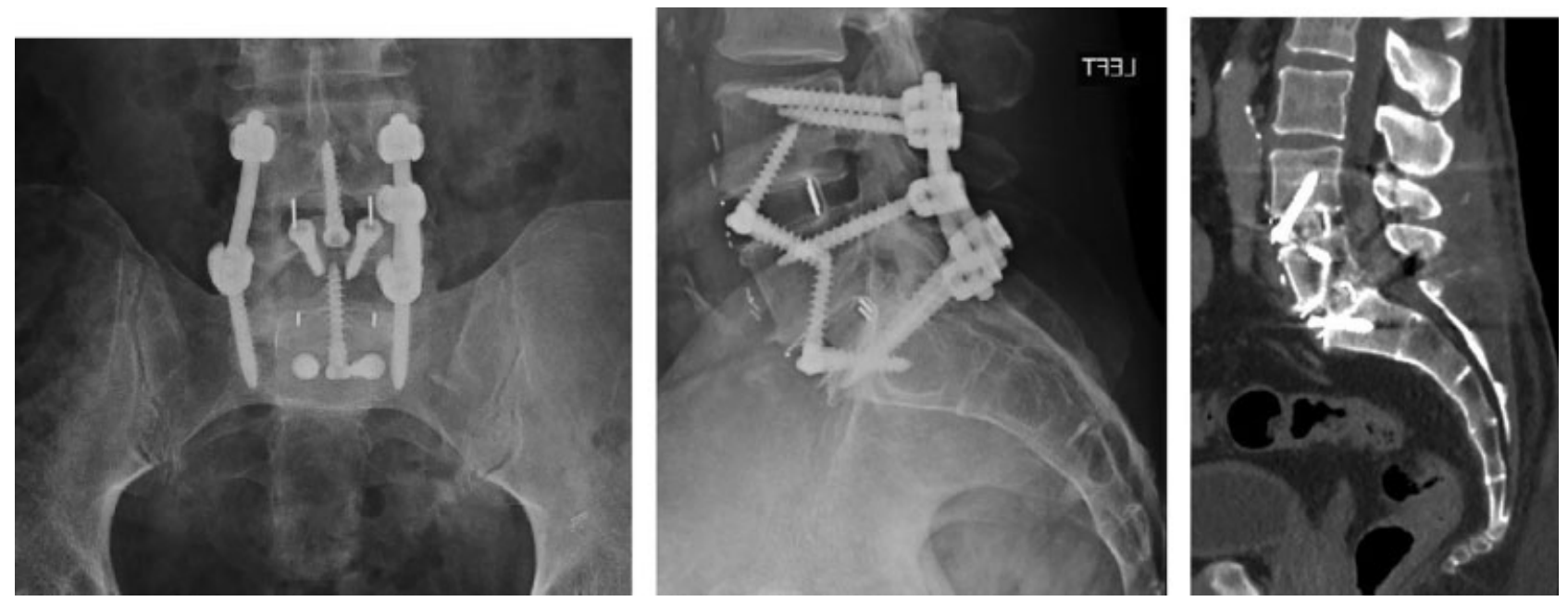

Fig. 1 Caso exemplo de ALIF a 2 níveis - L4-L5 e L5-S1-com caixas com 3 parafusos integrados e fixação transpedicular posterior percutânea. Radiografia em incidência anteroposterior, perfil e tomografia computadorizada mostra união (pontes ósseas intersomáticas L4-L5 e L5-S1) (paciente fez tomografia computadorizada do abdômen por outro motivo clínico).
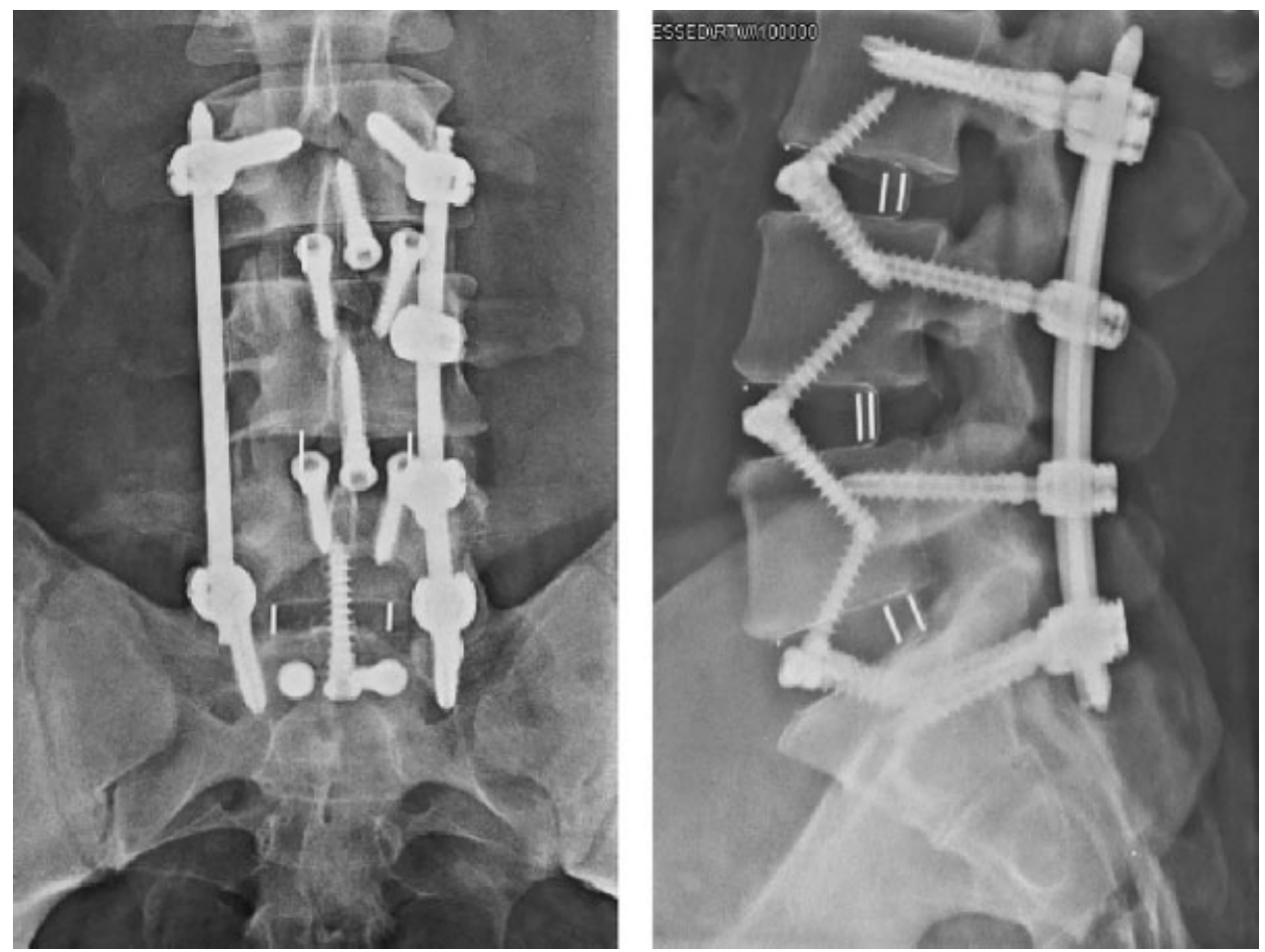

Fig. 2 Caso exemplo de ALIF a 3 níveis - L3-L4, L4-L5 e L5-S1-com caixas com 3 parafusos integrados e fixação transpedicular posterior percutânea. Radiografia em incidência anteroposterior e perfil.

foram: $14 \mathrm{~mm} / 8 \circ(\mathrm{n}=12)$ em L3-L4; $14 \mathrm{~mm} / 8 \circ(\mathrm{n}=12) \mathrm{e}$ $16 \mathrm{~mm} / 8 \circ(\mathrm{n}=12)$ em L4-L5; e $14 \mathrm{~mm} / 12 \circ(\mathrm{n}=12)$ e 15 $\mathrm{mm} / 12 \circ(\mathrm{n}=12)$ em L5-S1. Os parâmetros intra e pósoperatórios das ALIF estão indicados na - Tabela 2.

Verificou-se uma melhoria estatisticamente significativa entre a avaliação pré-operatória e a avaliação no fim do tempo de seguimento em ambos os escores analisados (-Figs. 6 e 7 ). 0 índice Oswestry passou de 71,81 $\pm 7,22$ no pré-operatório para $24,75 \pm 7,82$ na avaliação no fim do tempo de seguimento ( $\mathrm{p}<0,001$ ), corresponde à descida média de 47,06 \pm 5,29 (3754). Por sua vez, a escala visual analógica da dor passou de $7,88 \pm 0,70$ para $2,44 \pm 0,87(\mathrm{p}<0,001)$, corresponde à des- cida média de 5,44 $\pm 0,61$ (5-7). Verificou-se uma correlação significativa direta entre ambos os escores tanto na avaliação pré-operatória (rho $=0,79, \mathrm{p}<0,001$ ) como na avaliação final (rho $=0,87, \mathrm{p}<0,001$ ). Os pacientes com déficits neurológicos prévios apresentavam escores pré-operatórios significativamente menos favoráveis (Oswestry de 74,40 \pm 6,44 e escala analógica de $8,20 \pm 0,77$ ) em comparação com os neurologicamente intactos (Oswestry de 70,64 \pm 7,31 e escala analógica de $7,73 \pm 0,62, p=0,05$ e $p=0,016$, respetivamente). Os pacientes com fatores de risco apresentaram de forma significativa valores do índice Oswestry e da escala visual analógica pré-operatórios e finais menos favoráveis em comparação com 
144 ALIF multinível combinada com estabilização posterior em discopatia Moura et al.

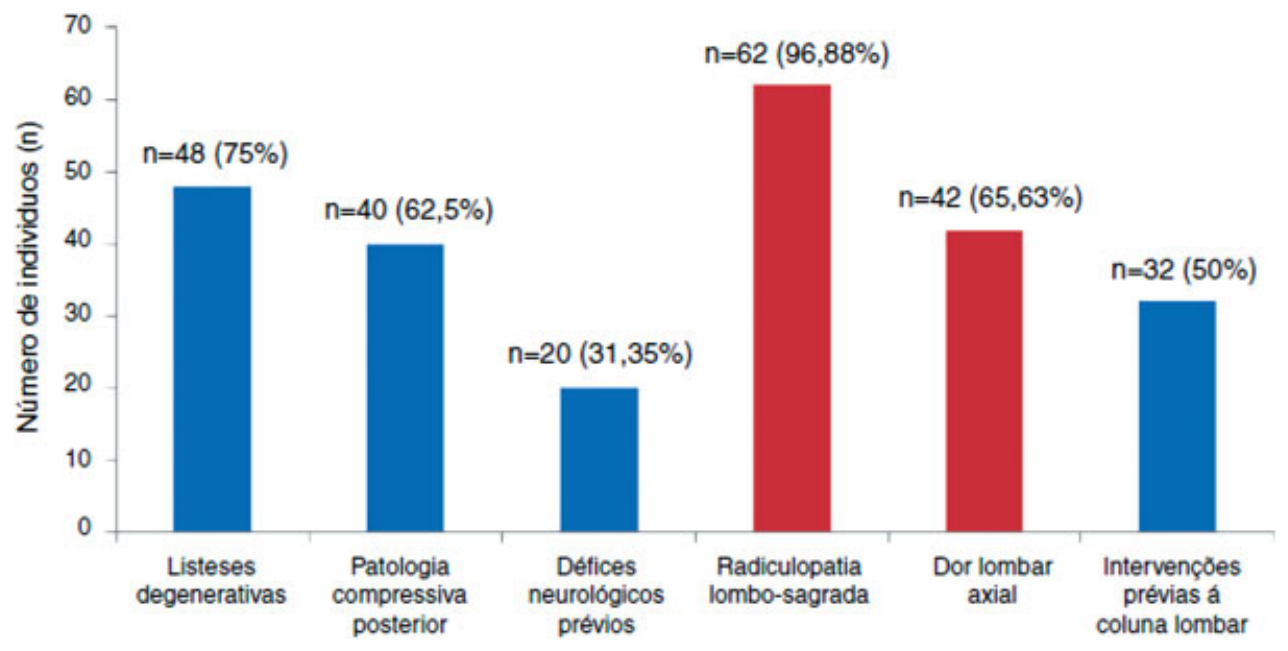

Fig. 3 Distribuição das indicações cirúrgicas e sintomatologia demonstrada.

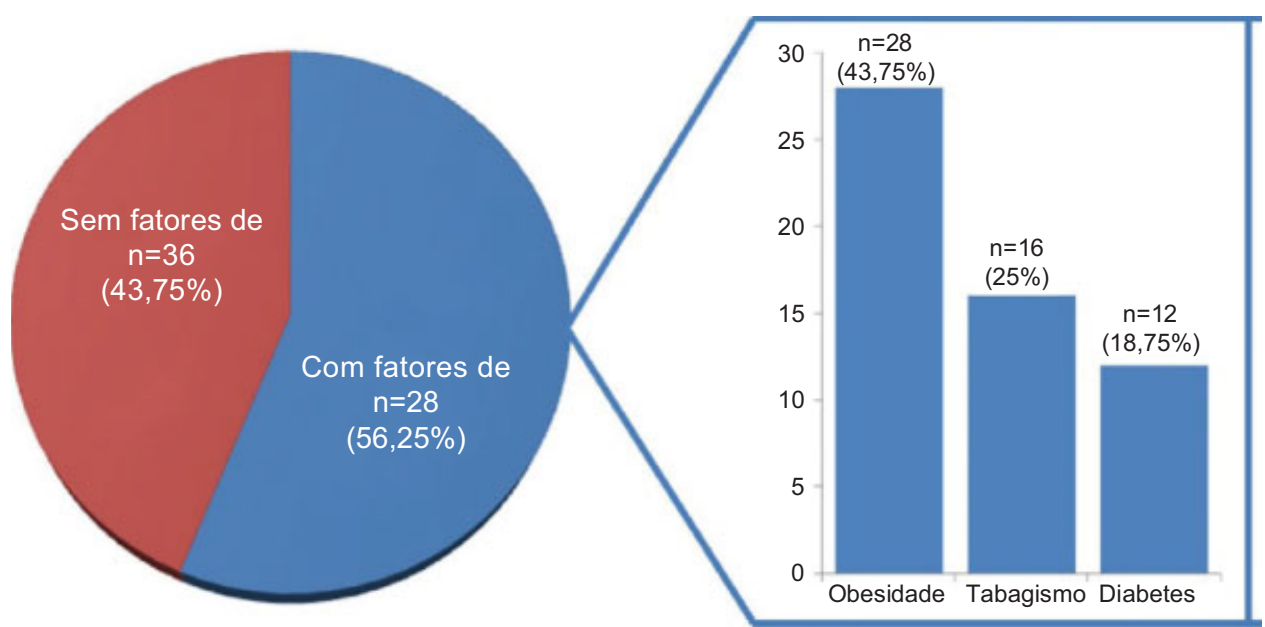

Fig. 4 Distribuição dos fatores de risco de não união da artrodese.

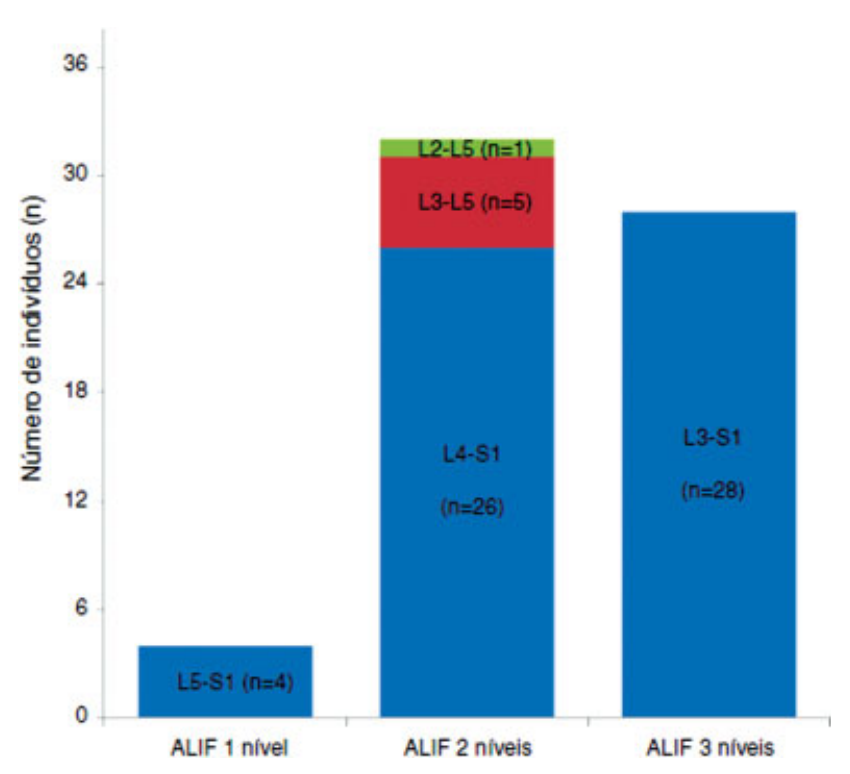

Fig. 5 Distribuição dos segmentos intervertebrais operados. Abreviações: ALIF, artrodese intersomática lombar anterior.
Tabela 2 Parâmetros intra e pós-operatórios das cirurgias ALIFs

\begin{tabular}{|l|l|}
\hline Parâmetros & Desvio-padrão \\
\hline Idade & $53,63 \pm 9,47$ anos \\
\hline Duração cirurgia das ALIFs & $105,63 \pm 24,49$ minutos \\
\hline $\begin{array}{l}\text { Perda sanguínea } \\
\text { ntraoperatória das ALIFs }\end{array}$ & $96,88 \pm 60,99 \mathrm{~mL}$ \\
\hline $\begin{array}{l}\text { Tempo de internamento } \\
\text { das ALIFs }\end{array}$ & $4,25 \pm 0,98$ dias \\
\hline
\end{tabular}

Abreviações: ALIF, artrodese intersomática lombar anterior.

os pacientes sem qualquer fator de risco identificado ( - Tabela 3). Ao analisarmos cada fator de risco separadamente, identificaram-se escores significativamente menos favoráveis nos obesos, nos fumantes, nos diabéticos e nos pacientes com cirurgias prévias à coluna lombar. Verificou-se ainda que os pacientes com patologia lombar posterior concomitante em que foi efetuada lamino-foraminectomia e artrodese 

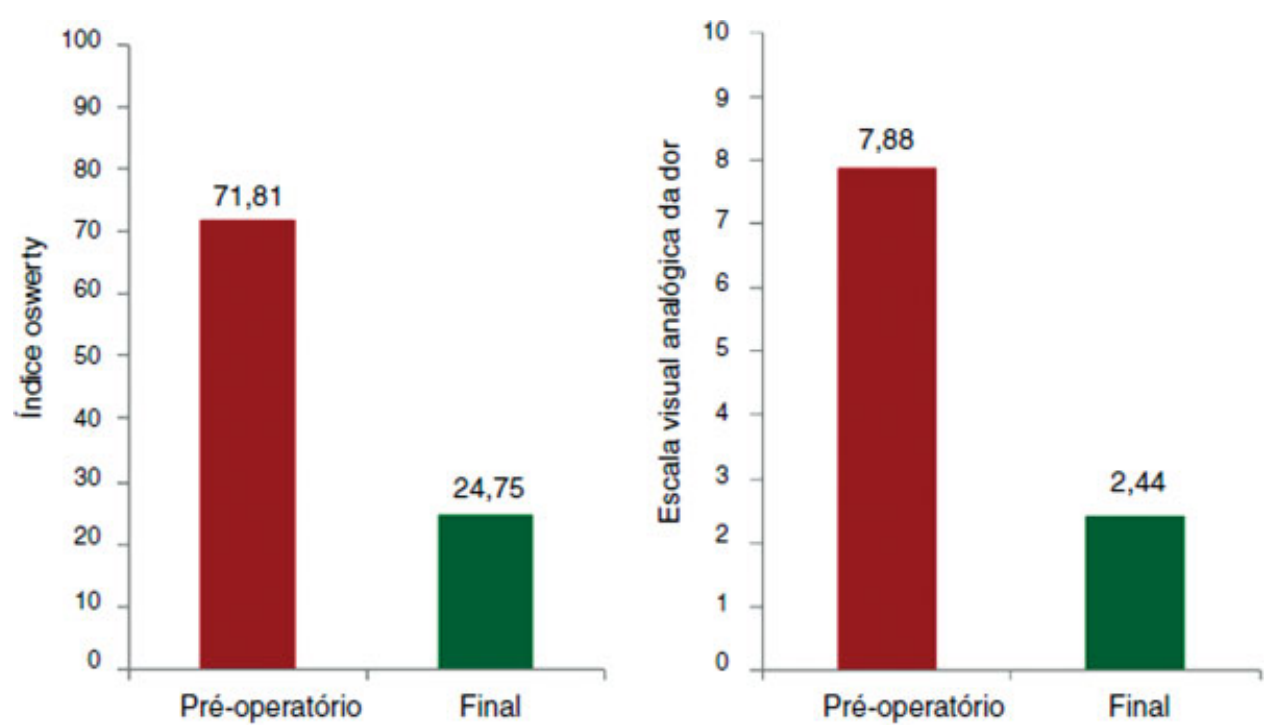

Fig. 6 Distribuição dos escores clínico-funcionais índice Oswestry e escala visual analógica da dor na avaliação no pré-operatório e na avaliação no fim do tempo de seguimento.

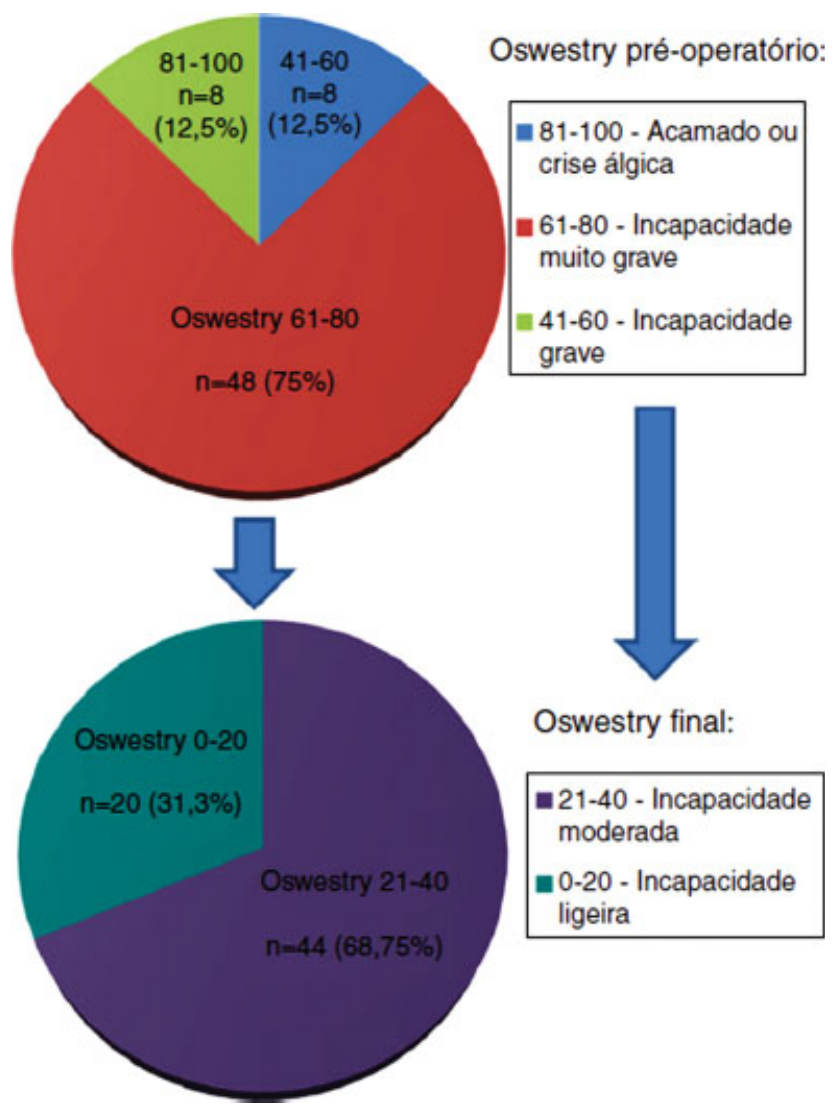

Fig. 7 Distribuição dos graus de incapacidade do índice Oswestry no pré-operatório e na avaliação no fim do tempo de seguimento.

posterolateral apresentaram também escores significativamente menos favoráveis (-Tabela 3). Verificou-se uma tendência para os pacientes com déficits neurológicos prévios apresentarem uma melhoria média dos escores clínico-funcionais mais acentuada, sem significado estatístico. Foi identificada uma correlação significativa no sentido direto entre o número de fatores de risco que cada indivíduo apresenta e os índices Oswestry no pré-operatório ( $r$ ho $=0,67, \mathrm{p}<0,001$ ) e final (rho $=0,79, \mathrm{p}<0,001$ ) e as escalas visuais analógicas préoperatória (rho $=0,39, \mathrm{p}=0,001$ ) e final (rho $=0,58$, $\mathrm{p}<0,001$ ). Foram ainda confirmadas correlac,ões significativas no sentido direto do número de níveis operados com a duração média da cirurgia (rho $=0,86, \mathrm{p}<0,001,1$ nível $=50,00$ $\pm 12,00 ; \quad 2$ níveis $=94,38 \pm 11,76 ; \quad 3$ níveis $=126,43$ $\pm 24,49$ minutos) e a perda sanguínea média (rho $=0,52$, $\mathrm{p}<0,001,1$ nível $=25,00 \pm 5,00 ; 2$ níveis $=103,13 \pm 81,75$; 3 níveis $=100,00 \pm 13,61 \mathrm{~mL}$ ). Além disso, a melhoria média de ambos os escores demonstrou uma correlação significativa no sentido direto com o número de níveis operados (rho $=0,40, \mathrm{p}=0,001)$, verificou-se a melhoria mais acentuada nos ALIFs de três níveis (-Tabela 4 ).

As complicações nas ALIFs foram apenas três infeções superficiais da ferida cirúrgica $(4,69 \%)$ e duas pequenas deiscências da ferida operatória (3,13\%). Não ocorreram complicações major ou fatais, tais como laceração ou trombose de grandes vasos, ou qualquer complicação intraoperatória. Não se verificou qualquer caso de hematoma retroperitoneal, hérnia incisional abdominal, ejaculação retrógrada ou disfunção eréctil. Não ocorreram complicações nas intervenções de estabilização posterior feitas num segundo tempo operatório. Assim, a taxa global de complicações foi de 7,82\% e a taxa de complicações major de 0\%. Não se identificou também qualquer caso de não união, migração dos implantes ou desenvolvimento de doença do disco adjacente no tempo de seguimento. $\mathrm{O}$ grupo de pacientes com complicações apresentava uma idade média significativamente superior $(64,50 \pm 4,81)$ ao grupo que não teve complicações $(52,07 \pm 8,95)(p<0,001)$.

\section{Discussão}

Consideramos que a ALIF combinada com fixação ou artrodese posterolateral é uma intervenção cirúrgica que garante uma coluna lombar mais sólida, estável, resistente e duradoura, é 
Tabela 3 Avaliação clínico-funcional em vários subgrupos e suas diferenças

\begin{tabular}{|c|c|c|c|c|c|c|}
\hline & $\begin{array}{l}\text { Oswestry } \\
\text { pré-operatório }\end{array}$ & Oswestry final & $\begin{array}{l}\text { Descida média } \\
\text { do Oswestry }\end{array}$ & $\begin{array}{l}\text { EVA da dor } \\
\text { pré-operatória }\end{array}$ & $\begin{array}{l}\text { EVA da } \\
\text { dor final }\end{array}$ & $\begin{array}{l}\text { Descida média } \\
\text { da escala visual } \\
\text { analógica da dor }\end{array}$ \\
\hline $\begin{array}{l}\text { Com fatores } \\
\text { de risco }\end{array}$ & $75,44 \pm 5,92$ & $30,22 \pm 3,85$ & $45,22 \pm 5,23$ & $8,11 \pm 0,75$ & $2,89 \pm 0,57$ & $5,22 \pm 0,42$ \\
\hline $\begin{array}{l}\text { Sem fatores } \\
\text { de risco }\end{array}$ & $67,14 \pm 6,00$ & $17,71 \pm 5,68$ & $49,43 \pm 4,42$ & $7,57 \pm 0,50$ & $1,86 \pm 0,85$ & $5,71 \pm 0,71$ \\
\hline Valor $p$ & $<0,001^{\mathrm{a}}$ & $<0,001^{\mathrm{a}}$ & $0,003^{a}$ & $0,03^{a}$ & $<0,001^{\mathrm{a}}$ & $0,002^{\mathrm{a}}$ \\
\hline Obesos & $77,00 \pm 5,16$ & $30,71 \pm 2,35$ & $46,29 \pm 5,44$ & $8,14 \pm 0,65$ & $2,86 \pm 0,36$ & $5,29 \pm 0,46$ \\
\hline Não obesos & $67,78 \pm 5,91$ & $20,11 \pm 7,43$ & $47,67 \pm 5,17$ & $7,67 \pm 0,68$ & $2,11 \pm 1,01$ & $5,56 \pm 0,69$ \\
\hline Valor $p$ & $<0,001^{\mathrm{a}}$ & $<0,001^{\mathrm{a}}$ & 0,384 & $0,007^{\mathrm{a}}$ & $<0,001^{a}$ & 0,127 \\
\hline Fumantes & $75,00 \pm 7,30$ & $29,75 \pm 4,97$ & $45,25 \pm 4,97$ & $8,00 \pm 0,73$ & $3,00 \pm 0,73$ & 5,00 \\
\hline Não fumantes & $70,75 \pm 6,94$ & $23,08 \pm 7,92$ & $47,67 \pm 5,30$ & $7,83 \pm 0,69$ & $2,25 \pm 0,84$ & $5,58 \pm 0,65$ \\
\hline Valor $p$ & $0,043^{a}$ & $0,002^{a}$ & 0,17 & 0,416 & $0,005^{a}$ & $<0,001^{\mathrm{a}}$ \\
\hline Diabéticos & $80,67 \pm 4,38$ & $31,33 \pm 0,98$ & $49,33 \pm 3,45$ & $8,33 \pm 0,49$ & 3,00 & $5,33 \pm 0,49$ \\
\hline Não diabéticos & $69,77 \pm 6,12$ & $23,23 \pm 7,92$ & $46,54 \pm 5,53$ & $7,77 \pm 0,70$ & $2,31 \pm 0,92$ & $5,46 \pm 0,64$ \\
\hline Valor $p$ & $<0,001^{\mathrm{a}}$ & $<0,001^{\mathrm{a}}$ & 0,097 & $0,011^{\mathrm{a}}$ & $0,007^{a}$ & 0,628 \\
\hline Cirurgia primária & $68,13 \pm 8,01$ & $19,63 \pm 7,53$ & $48,50 \pm 5,05$ & $7,50 \pm 0,51$ & $1,88 \pm 0,79$ & $5,63 \pm 0,71$ \\
\hline $\begin{array}{l}\text { Cirurgias prévias à } \\
\text { coluna vertebral }\end{array}$ & $75,50 \pm 3,70$ & $29,88 \pm 3,64$ & $45,63 \pm 5,20$ & $8,25 \pm 0,67$ & $3,00 \pm 0,51$ & $5,25 \pm 0,44$ \\
\hline Valor $p$ & $<0,001^{a}$ & $<0,001^{a}$ & $0,013^{a}$ & $<0,001^{\mathrm{a}}$ & $<0,001^{a}$ & $0,023^{a}$ \\
\hline $\begin{array}{l}\text { Com déficits } \\
\text { neurológicos }\end{array}$ & $74,40 \pm 6,44$ & $25,20 \pm 7,06$ & $49,20 \pm 3,97$ & $8,20 \pm 0,77$ & $2,40 \pm 0,82$ & $5,80 \pm 0,77$ \\
\hline $\begin{array}{l}\text { Sem déficits } \\
\text { neurológicos }\end{array}$ & $70,64 \pm 7,31$ & $24,55 \pm 8,21$ & $46,09 \pm 5,56$ & $7,72 \pm 0,62$ & $2,45 \pm 0,90$ & $5,27 \pm 0,45$ \\
\hline Valor $p$ & 0,907 & 0,058 & 0,062 & $0,016^{a}$ & 0,900 & $0,004^{a}$ \\
\hline $\begin{array}{l}\text { Com compressão } \\
\text { posterior }\end{array}$ & $73,90 \pm 7,31$ & $25,30 \pm 8,63$ & $48,60 \pm 5,48$ & $8,20 \pm 0,61$ & $2,60 \pm 0,93$ & $5,60 \pm 0,67$ \\
\hline $\begin{array}{l}\text { Sem compressão } \\
\text { posterior }\end{array}$ & $68,33 \pm 5,65$ & $23,83 \pm 6,31$ & $44,50 \pm 3,86$ & $7,33 \pm 0,48$ & $2,17 \pm 0,71$ & $5,17 \pm 0,38$ \\
\hline Valor $p$ & $0,003^{a}$ & 0,315 & $0,002^{a}$ & $<0,001^{a}$ & $0,022^{a}$ & $0,006^{a}$ \\
\hline
\end{tabular}

${ }^{a}$ Valores p com significância estatística. EVA, escala de valor analógica.

Tabela 4 Grau de melhoria ou descida média dos escores clínicofuncionais de acordo com o número de níveis operados entre a avaliação no pré-operatório e a avaliação mais recente

\begin{tabular}{|l|l|l|}
\hline \multirow{2}{*}{$\begin{array}{l}\text { Número de níveis } \\
\text { operados }\end{array}$} & \multicolumn{2}{|l|}{$\begin{array}{l}\text { Melhorias médias dos scores } \\
\text { clínico-funcionais }\end{array}$} \\
\cline { 2 - 3 } & Oswestry & $\begin{array}{l}\text { Escala visual } \\
\text { analógica da dor }\end{array}$ \\
\hline 1 & $40,00 \pm 1,11$ & $5,00 \pm 1,12$ \\
\hline 2 & $46,13 \pm 5,53$ & $5,25 \pm 0,44$ \\
\hline 3 & $49,14 \pm 4,16$ & $5,71 \pm 0,71$ \\
\hline
\end{tabular}

um procedimento particularmente importante em pacientes jovens, em que o restauro do balanço sagital e das cargas fisiológicas sobre as colunas de Dennis permitem diminuir a evoluc,ão para doença do disco adjacente, sobrecarga da coluna posterior e evolução artrósica precoce. ${ }^{2,4-6,8}$ As recentes técnicas de ALIF instrumentada têm resultados comprovados na literatura, verifica-se melhoria significativa dos escores clínico-funcionais após a intervenção cirúrgica, com taxas de união da artrodese acima de $90 \%$ e de complicações major inferior a $10 \%$. $3,8,10,16,17$

Apesar de boa estabilização anterior da ALIF instrumentada atual, entendemos que, sobretudo na presença de fatores de risco de não união, é importante complementar a construção com estabilização transpedicular posterior, de modo a obter uma construção mais estável e assim promover ao máximo a fusão intersomática desejada e diminuir o risco acrescido de não união desses casos. No entanto, esse tema é controverso e existem atualmente resultados contraditórios na literatura. Alguns trabalhos demonstram que a ALIF instrumentada stand-alone sem estabilização posterior não tem diferenças significativas em termos de resultados clínico-funcionais e radiográficos em comparação com a artrodese lombar circunferencial, pode-se assim evitar uma segunda cirurgia nos casos em que não há necessidade de descompressão posterior. ${ }^{3,6,7,9-11,18,19}$ No entanto, outros 
estudos demonstram taxas de união superiores para as ALIFs instrumentadas combinadas com fixação posterior e que, apesar de a fixação da ALIF com placa anterior ou com parafusos integrados na caixa aumentar de forma significativa a estabilidade da construção, essa continua a ser inferior à obtida com a instrumentação posterior. ${ }^{20-25}$ Além disso, a estabilidade adicional oferecida pela fixação ou artrodese posterior pode provavelmente contribuir para diminuir a intensidade ou mesmo evitar o aparecimento de sintomas na presença duma não união da ALIF, permite que essas não uniões sejam bem toleradas ou mesmo assintomáticas. Consideramos necessários mais estudos prospetivos aleatorizados de grande dimensão de modo a comprovar a eficácia e segurança das caixas de ALIF instrumentadas sem necessidade de estabilização posterior.

A solidez da montagem anterior e posterior e o potencial osteoindutivo da BMP-2 na caixa intersomática poderão ser responsáveis por não se registrar qualquer não união nesta amostra, mesmo perante uma quantidade substancial de pacientes com fatores de risco de não união e perante metade da amostra apresentar cirurgias prévias à coluna lombar. 0 uso de Infuse ${ }^{\circledR}$ (Medtronic, Fridley, MN, USA) permite também evitar a morbilidade e eventuais complicações com origem na colheita de autoenxerto da crista ilíaca. ${ }^{3,10}$ Apesar dos pacientes com não uniões de PLIFs prévios terem apresentado escores clínico-funcionais menos favoráveis, a revisão através de outra via de abordagem (via anterior) e a ALIF permitiram obter resultados bastante satisfatórios. ${ }^{2,26}$

A melhoria clínico-funcional crescente de acordo com a intervenção num número superior de segmentos intervertebrais deve ser analisada com precaução na medida em que o grupo da ALIF uninível está limitado a quatro pacientes, podem os resultados estar enviesados por reduzida dimensão desse grupo em comparação com as ALIFs de 2 e 3 níveis e consequente perda de poder estatístico. Ainda assim, consideramos que esses resultados poderão ser justificados devido à ALIF de 2 e 3 níveis (corresponde a 93,75\%) permitir não só tratar esses segmentos individualmente, mas também evitar uma eventual deterioração clínica por doença do disco adjacente ao garantir de forma mais fidedigna a restauração da lordose lombar e balanço sagital fisiológicos em comparação com a ALIF a um nível. A melhoria clínico-funcional verificada nas ALIFs multinível pode também permitir afirmar a reduzida morbilidade da via anterior retroperitoneal da coluna lombar na extensão da intervenção a vários segmentos, garante que a intervenção multinível não prejudique negativamente a recuperação clínico-funcional mesmo tendo em conta que essas têm duração cirúrgica e perdas hemorrágicas superiores às ALIF uninível.

Apesar do risco de complicações potencialmente fatais associadas à via anterior abdominal retroperitoneal, consideramos que a sua execução e responsabilização (tanto na abertura como tratamento de eventuais complicações vasculares, encerramento e seguimento de qualquer complicação no pós-operatório relacionada com a via de abordagem) por um cirurgião com vasta experiência nessa abordagem poderá ser um dos fatores importantes para a reduzida taxa de complicações verificada nesta amostra. ${ }^{2,3,6,8,16,27-30}$ Além das carac- terísticas de reduzida morbilidade da via anterior retroperitoneal, a experiência do cirurgião nessa abordagem permite poupar tempo de cirurgia e diminuir perdas hemorrágicas, o que poderá também contribuir para a reduzida taxa de complicações, curtos períodos de internamento e melhoria clínico-funcional dos pacientes neste estudo. ${ }^{28,30}$

Em suma, consideramos que os resultados clínico-funcionais e radiográficos muito satisfatórios obtidos neste estudo se devem às vantagens biomecânicas da ALIF instrumentada combinada com estabilização posterior, ao uso frequente da ALIF multinível e às suas vantagens da restauração mais eficaz da lordose lombar, à técnica cirúrgica tanto da via de abordagem como na cirurgia da coluna e às caixas intersomáticas com parafusos integrados e preenchidas com matriz óssea e BMP-2.

Este estudo prospetivo tem como principal vantagem face a estudos semelhantes a uniformidade da aplicação do mesmo protocolo terapêutico a todos os pacientes e o fato de todos terem sido operados pelos mesmos cirurgiões, o que permite diminuir consideravelmente eventuais vieses por variações no tratamento. Como principais limitações destacamos este estudo não ser aleatorizado nem cego e tratar-se duma amostra de conveniência e heterogênea em termos de dimensão de grupos.

\section{Conclusão}

A biomecânica favorável da ALIF instrumentada e combinada com estabilização posterior é uma opção terapêutica satisfatória na discopatia degenerativa uni ou multinível dos segmentos L3-L4, L4-L5 e L5-S1, associada ou não a patologia lombar compressiva posterior, mesmo em situações de presença significativa de fatores de risco de não união e cirurgias prévias da coluna lombar, garante resultados clínico-funcionais e radiográficos muito satisfatórios e uma reduzida taxa de complicações em médio prazo.

Conflitos de interesse

Os autores declaram não haver conflitos de interesse.

\section{Referências}

1 Phan K, Thayaparan GK, Mobbs RJ. Anterior lumbar interbody fusion versus transforaminal lumbar interbody fusion-systematic review and meta-analysis. Br J Neurosurg 2015;29(05): 705-711

2 Rao PJ, Loganathan A, Yeung V, Mobbs RJ. Outcomes of anterior lumbar interbody fusion surgery based on indication: a prospective study. Neurosurgery 2015;76(01):7-23

3 Burkus JK, Gornet MF, Schuler TC, Kleeman TJ, Zdeblick TA. Six-year outcomes of anterior lumbar interbody arthrodesis with use of interbody fusion cages and recombinant human bone morphogenetic protein-2. J Bone Joint Surg Am 2009;91(05):1181-1189

4 Truumees E, Majid K, Brkaric M. Anterior lumbar interbody fusion in the treatment of mechanical low back pain. Semin Spine Surg 2008;20(02):113-125

5 Mummaneni PV, Haid RW, Rodts GE. Lumbar interbody fusion: state-of-the-art technical advances. Invited submission from the Joint Section Meeting on Disorders of the Spine and Peripheral Nerves, March 2004. J Neurosurg Spine 2004;1(01):24-30 
6 Strube P, Hoff E, Hartwig T, Perka CF, Gross C, Putzier M. Standalone anterior versus anteroposterior lumbar interbody singlelevel fusion after a mean follow-up of 41 months. J Spinal Disord Tech 2012;25(07):362-369

7 Zhang JD, Poffyn B, Sys G, Uyttendaele D. Are stand-alone cages sufficient for anterior lumbar interbody fusion? Orthop Surg 2012;4(01):11-14

8 Udby PM, Bech-Azeddine R. Clinical outcome of stand-alone ALIF compared to posterior instrumentation for degenerative disc disease: A pilot study and a literature review. Clin Neurol Neurosurg 2015;133:64-69

9 Kim JS, Kim DH, Lee SH, Park CK, Hwang JH, Cheh G, et al. Comparison study of the instrumented circumferential fusion with instrumented anterior lumbar interbody fusion as a surgical procedure for adult low-grade isthmic spondylolisthesis. World Neurosurg 2010;73(05):565-571

10 Lammli J, Whitaker MC, Moskowitz A, Duong J, Dong F, Felts L, et al. Stand-alone anterior lumbar interbody fusion for degenerative disc disease of the lumbar spine: results with a 2-year follow-up. Spine 2014;39(15):E894-E901

11 Madan SS, Boeree NR. Comparison of instrumented anterior interbody fusion with instrumented circumferential lumbar fusion. Eur Spine J 2003;12(06):567-575

12 Glassman SD, Anagnost SC, Parker A, Burke D, Johnson JR, Dimar JR. The effect of cigarette smoking and smoking cessation on spinal fusion. Spine 2000;25(20):2608-2615

13 Pilitsis JG, Lucas DR, Rengachary SS. Bone healing and spinal fusion. Neurosurg Focus 2002;13(06):e1

14 Fairbank JC, Pynsent PB. The Oswestry Disability Index. Spine 2000;25(22):2940-2952, discussion 2952

15 Freyd M. The graphic rating scale. J Educ Psychol 1923;43:83-102

16 Sasso RC, Kitchel SH, Dawson EG. A prospective, randomized controlled clinical trial of anterior lumbar interbody fusion using a titanium cylindrical threaded fusion device. Spine 2004;29(02): 113-122, discussion 121-2

17 Pavlov PW, Meijers H, van Limbeek J, Jacobs WC, Lemmens JA, Obradov-Rajic M, et al. Good outcome and restoration of lordosis after anterior lumbar interbody fusion with additional posterior fixation. Spine 2004;29(17):1893-1899, discussion 1900

18 Choi KC, Ryu KS, Lee SH, Kim YH, Lee SJ, Park CK. Biomechanical comparison of anterior lumbar interbody fusion: stand-alone interbody cage versus interbody cage with pedicle screw fixation - a finite element analysis. BMC Musculoskelet Disord 2013; $14: 220$
19 Cain CM, Schleicher P, Gerlach R, Pflugmacher R, Scholz M, Kandziora F. A new stand-alone anterior lumbar interbody fusion device: biomechanical comparison with established fixation techniques. Spine 2005;30(23):2631-2636

20 McCarthy MJH, Ng L, Vermeersch G, Chan D. A radiological comparison of anterior fusion rates in anterior lumbar interbody fusion. Global Spine J 2012;2(04):195-206

21 Anjarwalla NK, Morcom RK, Fraser RD. Supplementary stabilization with anterior lumbar intervertebral fusion-a radiologic review. Spine 2006;31(11):1281-1287

22 El Masry MA, Badawy WS, Rajendran P, Chan D. Combined anterior interbody fusion and posterior pedicle screw fixation in patients with degenerative lumbar disc disease. Int Orthop 2004;28(05):294-297

23 Oxland TR, Lund T. Biomechanics of stand-alone cages and cages in combination with posterior fixation: a literature review. Eur Spine J 2000;9(Suppl 1):S95-S101

24 Tzermiadianos MN, Mekhail A, Voronov LI, Zook J, Havey RM, Renner SM, et al. Enhancing the stability of anterior lumbar interbody fusion: a biomechanical comparison of anterior plate versus posterior transpedicular instrumentation. Spine 2008;33 (02):E38-E43

25 Beaubien BP, Freeman AL, Turner JL, Castro CA, Armstrong WD, Waugh LG, et al. Evaluation of a lumbar intervertebral spacer with integrated screws as a stand-alone fixation device. J Spinal Disord Tech 2010;23(05):351-358

26 Gertzbein SD, Hollopeter MR, Hall S. Pseudarthrosis of the lumbar spine. Outcome after circumferential fusion. Spine 1998;23(21): 2352-2356, discussion 2356-7

27 Chiriano J, Abou-Zamzam AM Jr, Urayeneza O, Zhang WW, Cheng $\mathrm{W}$. The role of the vascular surgeon in anterior retroperitoneal spine exposure: preservation of open surgical training. J Vasc Surg 2009;50(01):148-151

28 Mobbs RJ, Phan K, Daly D, Rao PJ, Lennox A. Approach-related complications of anterior lumbar interbody fusion: results of a combined spine and vascular surgical team. Global Spine J 2016;6 (02):147-154

29 Wood KB, Devine J, Fischer D, Dettori JR, Janssen M. Vascular injury in elective anterior lumbosacral surgery. Spine 2010;35(9, Suppl):S66-S75

30 Quraishi NA, Konig M, Booker SJ, Shafafy M, Boszczyk BM, Grevitt MP, et al. Access related complications in anterior lumbar surgery performed by spinal surgeons. Eur Spine J 2013;22(Suppl 1): S16-S20 